\title{
TENDENCIAS EMPRESARIALES ACTUALES Y SU IMPACTO EN LA GESTIÓN HUMANA
}

\section{Por Cristhian Guillermo Naranjo Herrera*}

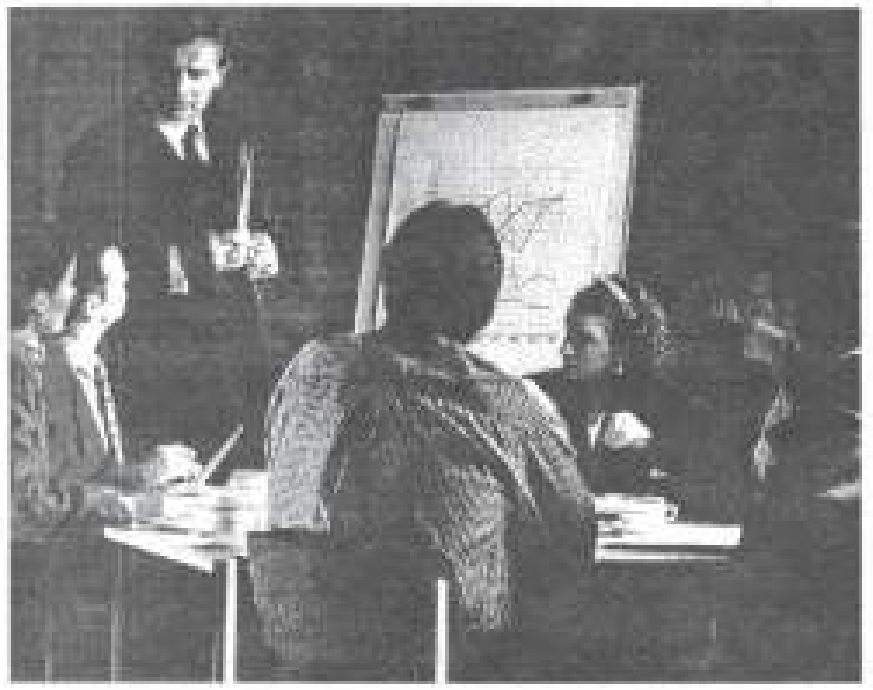

17 ivimos una época de profundos y acelerados cambios. Se nos pide ier y hsoer más con menos recursos y adaptarnos al un entomo de mayot eomplejidad, exigencia y diversidad. No-se trata de la parafernalia que un susevo milenio sucle generar en la raza humana, por el contrario, es la confirmación del escenario competitivo y desafiante que desde déeadas anteriores, pero en partieular en los años neventa nos ha oxrreggondido en suerte pressenciar, resultando imperioso el análisis y comprensión del contexto en que nos movemas. con un criterio prospectivo que permita no sollo sobrevivir en un periodo puntual, sino proyectar la arpanización para que evolucione al ritmo requerido, la cual nos leva a considerar las tendencias empresariales dominanted. Nos ocuparemos entooces, de manca breve, pers cijala afortunada, de describer algunas de las caracteristicas y tendencias que se encuentran en el ruundo empresarial para proceder en la seganda parte del articulo a establecer el efeeto que se desprende do las mismas para la función de pernosal, en urua a contrituir al mejoramsento de has áreas de Gestón Humami.

\section{TENDENCIAS}

Ias cosas no sun cono solian ser. Heanos pasado del merundo local a la globalizacion; del control de dichos meteades por parte de los productores al reinsdo de los elientes; los faetores competitivos

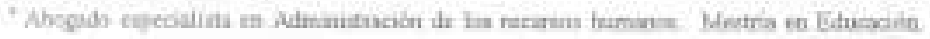


tradicionales como el precio y la calidad, siguen siendo necesarios pero insuficientes $y$ asistimos a la emergencia de nuevos factores eome la adaptabilidad, la innoysción y el tiempo do respuesta, eritre otros, la couposición de is fueres faboral exige mano de obra califieada, contempla personal jowen pero experto, la participación de la mujer ts caís vez mayor, incrementàndoue el tumaño y complejidad del metcado de trabajo: Is rigidez de las organizaciones y de las relaciones laborales ha cedido en fovor de la flexibilidat, los estilos de dirección y los sisteman de condrol se han repilanteado de mancra dramática, d trabajo ea equipo enotinta desplazando al desempeño individual; el avance en las tecnologias de información y Ins teleconumicaciones impucta y modifica la estruclura de la empreas y la manera de realizat It prestación de kos servicios, en sintesis, el panorama actual sisth mucho del que fan solo hace unos cuantis afíca percibiamas, söcndo apenas lógieo que nuestros paradigmas y las acciones que desarroullanos sear sastancialmente diferentes a las que hahitualmeste nos hàn acourpañado.

\section{La orientación al cliente}

Desde luego que la existencia misusa de una organización y sus postrilidades de crocimiento están directamente reiaciónadas con su capacidad de conacguir y maniener clientex. Lo metrior no representa nada nuevo. pero si lo es el enfoque y la importaseia del efiente. Veamos: En el pasedo el clienie texia que conformarse con lo que la emprean quisicien darle, atan en detrimento de la calidad y of servició, era. la empresa quien imponia sut condiciones $y$ ante la escasez de alternativas ef cliente quodabs maniatado y cautivo. Hoy, el clieate euenta eon un abunika amplio para eseoger a quièn le cempora, posee un buen conocimiento del mercado, el poder de los compradores ha aumetitado a la par que el de los provecdores se ha reducido, es el cliente el qae impone las condiciones, al punto que el disetio miano do los productow y servicios, la produceion de los bienes, algunos sistemss de control y la estructurn organizacional se construyen y administran pensanido en las necesidades y expectativas del cliente, otorgandole a éste una participación dirceta e ineluse un segmeato importante en la toma de decisiones de la empeesa. - por ejemplo coloeando al clicnte en la cima do is tradicional pirlmide y junin a ellos los empleados que les sirven y con los que más entra en contacto, mientras que Ins firectivos cierran el cielo y se conciben como apoyo o al servicie de dicha estruetura o de las distinsas unidader de servició
- El cliente es el rey y todos nuestron esfiserzos se cacantinan a conservarlo y motivar un inerements on el volumen y ealidad de nuestras teiaciones con é. La calidad total, los sistemas de producción justo a tiempo y la gerencis del servicio, eatre otros modelos umpliamente dilundidos y aceptadrxi, constituyen un buen ejemplo del enfoque centrado on el clisente.

\section{GLoBaLIzACióN}

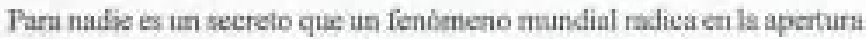
económica y comercial. Las fronteras y obotáculos desaparecen y de manera casi instantinea tenemos que eompetir en un mercado glohal que sustitoye al qparroquialo o loval que conocinanos y de alguna maneen controlabamos. El auge de las exportaciones, la necesidad de reconvertir muestras industrias, la presencia de ura nunserosa y sgresiva competencia, las exigencias de ealidad porn pesetrar mereados, ef conoeimiento del eoncierto internacional, la conformación de bloques. la nocesodad impostergable de estabiecer dilisuas estrategicas, de repensar la visión estratégica del negocio, de ir a la vanguardia en lo relacionado eon la tecoologia, son todos sintomas y desaflens que plantea esta tendencia que, a no dudarlo, culminará por incluir aspeoioe politicos y legales, porque en el plano seciocultural es ya evidente Es apenas obvio onncluir que en este nuovo escenario se hace impresxindible una mentabidad mik amplia y abierta, orpanizaciones con mayor đinámica y propecciỏn, prandes inversiones en tecnologia, constante cuslificsción y eapacidad para integrar diversaz sulturas, comp rasgos spbresalientes

\section{COMPETITIVIDAD}

Con lo dicho hasta ahora, queda clare que ya no ommpetimos aen cnsay, sino globalmente; que nuestros competidares son de talla mundial; que al menor descuido se nos saen del mercado, que kne tactores clave han aumentado y se han diversificado, al punto que al cliente oo le besta con un producto de calidad que le satistaga, sino que trasoendiendo su satiefacción, se logre su enountamienis, lo que implica que cada experierseda sea adezas de grata, inolvidable y siempre genere el deseo de su repetición, por otra parte, se exige us buen producto o servicia peno al màs hajo precin posahle y que se afrezcan las debidas parantias de tal suette que une u otre sean confiables y, de 
sef posibie, daraderos. todo to cunl desemboca en la eficiencia y eficacia de nuestrios procesos. Al margen de estos facteres tridicionales de competitividad surgen otros camo los referidos al tiempo y la capacifad de adaplnción $e$ innovación de la empresa. ¿Para qุue sirve un exeelente producto o servicio si no se provee a tiempo? En la egilidad o rapidez eon que identifiquemos to qute el cliente desea o necesita $y$ el trempo que tardemas en responder a eliso poode estar la diferenuta en que realice sus negocios con nosotros o con la competeacia, mientras cque si no mejoenmos continuamente nuestros productos y servicios de tal formin que kas chientes perciban en ellos un tanyor valor, no faltana quien lo hage y nos sustituya, por lo que In capacidad de adaptamow a las exvencias del medio de manera continua, veloz, y de innovar, representian maevos factores competitivos de grin importansia, Ieniendo en cuenta que la empresan deberá ser lo suficientemente flexible para modificar sas productos y servicios acorde can lo solicitado por los clientes y en contra via de productos y servicios unifomes. o estandarizados sin opeibon de varianiter. En palabras sencillas, si producimas eepillos, no basta con una, dos o tres reforencias, aino que doberaos estar en capacidad de producit solure un modelo tásico, cuankos tipos se les ocurras a los elientes o requiera el merebdo. Este esqueran demanda olianivas estratégecas con proveedorell $y$ atras arganizaciones, inclusive aquellas que son competencia, puesto que es poeo probable que solos podamos ser los mejores nün cuando bastante tenemos que hacer al interior de las organizaciones en términos de la loenologia, el rectiseto de los procesos, la cualifieación del pernonal, teto obmo se verd posteriormente.

\section{NUEVOS TIPOS DE ORGANIZACIONES}

Los aspectos antes descritos hacen imperioso ajustar y erear nuevas estrueturas organizacionales. La cläsica organizzeción piranidal, con kafasis funcional, basada en departamentos y puestos de trahujo, el poder y la autoridad, viene cediendo ante la irrupeión de onganizaciones divisionales, eonglomerados y bolding, las organizaciones matriciales y particularmente con el auge de las organizaciones en red, las formas foderstivas. los grupos homegeneos (cluster organization). las unidedes de negocio y nuevas formas matricialos; Lo cierto es que urge disefiar organizsciones que den lugar al procesamiento, acesso y uso integrado de la información, fluyendo esta con rapidez en todos los niveles de la organización, mientras que en arns s la eficiencia y los resultados se tiende a organizaciones como sostiene Ricard searacterizadas por un mayor oomponente lateral, onientado a procesos, con limites erganizativos fluidos, tanto deatre de la organización (redell internns, formas federativas) como fuera de la mista (redes externas estables o dinamicas, otras foemas de cooperación oomo alinnzas, joint-ventures o agrupacioties de intetés económico), con un papel creciente de las tecnologias de la información y de la erganizaciōn, del trabajo eo equipos, con estructuras más planas y flexiblesb: (1) Sin einbargo, no basta con cambiar el creaniamamu o la estractura desde el pusto de viste formal o estetieo, sino que lo esencial radica en el eambio de mentalidad gerencial que corresponda efectivarnenic a la nueva estructurn, pries de nada sirve o, lo que es mis exacto, es contraproducente, contaf con una organización plana sí ac̀n pensamos y actuanos desde is perspectiva piramidal y
Do comprendemes los cambios on términes de conltol, participación, autenomia y jeratquin que ello impliea, siendo ineoneruentes, confundiende al personal y fomando docisiones para una emptesa que no es la diseriads y en un ambiente que mo eompartirk tas mismas, sfectande negativamente el elima laboral, la produetividad y las perspectivas de la empresh. En suma, se requiere de compromiso directivo y del conocimiento y adocuación de todos los niveles a is filosofin y exigencias de la aueva estructura. para que tenga oportunidad de funcionat óptimamente.

\section{LA ERA DE LA INFORMACIÓN Y EL CONOCIMIENTO}

Pricticamente no exiate en nuestros dias una sola empresa que no haga usodel computador Los avances tecoolagicos se suceden uno tras otro a un paso $\tan$ yertiginose que es fificil it a la par, ae ealcula que las techologias de la información se duplican cada 18 meses bien ses en cantidad o en velocidad, lo que en forma directamente proporcional gemera oportunidades de nuevor negocios o al meros el mejoramiento y variedad para efectuar ba ya existentes. Se concibe exisi como un pecado estaf por fueta de las ventajis que ofrece Internet, al ne contar con tana página web. descronocer el comercis electrúnioo y demás opciones que la rod afrece, cont una cobertura universal y a costos mucho más fuvorables que los que tradicionalmente maneja lo organización en áreas coms la pablicidad. sumando a lo assierior el que dicha red funciona 24 horgs al dia durante todos los dius del año.

En el contexto que bemos deserito resulta 
esencial in recolecebón, antilises y distribución de la información, camo insumo háaico para los procesos de toma de decisjones y como saporte para la realración exitosa de las metas de la instítución. Conocenos de abbra cómo la aulomaticación de los procesos los hace mäs eficiente, reduce sus cossos e incrementa la velocidad y capacidad de respoesta. Pero, no basta con tener y procesar la informacoón; se requiere que el personal desarmile nuevos aaberes y habilidades para que aptoveche al maximo sus posibilidades, ufilice adecuadamente las berramientas teenológieas y se adaple a los continuas cambios que esta àrea presenta. Las orpanizacinnes entoncer Jeben set rinteligentess y desarmollar su capocidad de aprendiaje porque de fo contranis quedarin a la sapa del mercado. La gestión del conocimiento toma ast una fuerza y valot prepeoderantes, imumpiendo tambien In noción del capital intelectual como mecantiumo de medicion y valaración de los activos intaagibles

El panotams prekentado tiene hondss fepercusiones en la Administracion del Talento Humano puesto gue si las circunstancias tàmbian o pueden llegar a hacerlo, tendri también que cambiar la gente a quiere maratenerse dentro del mereado laboral y progresar al interior de las arganizacioneh, mientrak que estas deberin repiantear Jos perfiles, las potiticas, ks roles, la manera de compensar, dirigir. syaluar, eecoger, fetener y dessurbilar a su personal, oamo factor oompetitrwo en esta nueva era. En la segunda parte de eate trabajo se analivaran los reton que las tendencias modernas planiean al àrea de Gestión Hemana.

Hasta nhora nos ocupamos de describir, de manera resumida, algunas de las tendencias actualen mas sobresalientes del mundo empresarial, eneno requisito previo para proceder laego a sbordar el impseto que las mismas generan en el Sistema de Oestión Humana Organizacional, tarea que nos llevara a la reviaión de la concepoón de la peraona y ei trahsjo dependiente y avalariade, la dirección estratégiea de los mal Hanakis recursos bumanos, el perfil de los responsables de la fanciobt de periomal, las pempectivas y roles del staff, In consecuencias en materis laboral de lis inmovaciones y avanoes tecnolbgicos, asi como de las tipos de organización emergentes, las competencins que se exigiran a la fuerza de trabajo, en fin, uan serie de cambias que a no dodar implican un replanteamiento de fondo en el Qué, Quieñes y Cóno Administrar el Personal en la Organizacóóe, representando complojiss y profundos desafins para la empresa miama. Sin mis preámbulos, examinemos los retes que lo unatado demanda.

\section{LA CONCEPCIÓN DEL SER HUMANO Y EL TRABAJO EN LA EMPRESA}

Durante muchos anos hemos dixefiado y organizado ef trabajo al interior de las organizaciones on base en el puesto de trabajo eaencialmente, dentro de una estruotura jorárquaca y funcional, de dende surgeo los manuales de funciones y procedimientos que le indican a los personas qué deben hacer, cömo hacerlo, para qué hacerin y limifan su eapacidad do scción y creatividad al establecer clara fronsteras, responsahilidaides y poder purn tomar decisiones. Observese ómo en las descripciones de eargos suele incluine un apartado gue se ocupa, a phari, de establocer la autonomia para la tima de deciaiones y la dependencia de un eargo (o persona, agregarlamos) superior. cronndo de un plumazo burreras a la liberted del personal y subordinindolo a quienes ocupan un lugar mats atto a lo largo de la cadena de mando, sin que necexariamente estos últimas posean la verdad reveladsé sean mis o mejor que quienes se ubican por ndebajos Con razón al ocuparse de este aspecto, Samdalio Gómez sostiene que alistamos coronando a tin kroyo que noe acaba sometiendo a todos. eet puesto de trabajow. Alrededor de el va a girar toda lu coguntiración, las póiticas de actuación y cada una de las pautas de eomportamieatoo (2). Bajo este esquema, por obvias razones, se hace necesaria ta cspecialización y la subdivisión do las tareas en su minima expresión posible - no en vano ef puesto de trabajo en la unidad banca de análisis del trabajo en la organización- reduciéndose la upoión de innoyar de trascender, de variar, en suma, atrapande a quien In descmpeña entre seuatro paredess para expresarlo do un modo gràficon.

Tradicisnaimente, la descripción y andisis de cargos es una füciỏn uignads a Gesticn Humana, por lo que aparece ol primer efeuto de las tendencias con relación al area de personal: Debe pasarae de las personss al servicio det eargo, al liseine del trahajo enfoeado al servicio de las personax, o loque es lo misma, DEL PUESTO DE TRABAJO, AL ÁREANATURAL DE TRABAJO,

La figidez del pueste de tratajo y la manera como eneasills a la uente se encuentra on coatra via de las tendenciss que hemos desarrollado, maxime en una èpoce en la que las persones reclaman, cois razbro. mayor participación, respeto, libertad y cenfianza. haciéndese necesario implementar un nuevo modelo que sgrupe cargos afines en unidades de mayor dimeasion que den luegr a la variedad, al trabajo integrado en vez del aislamiento individual del pacito de trabajo, a los 
equipos de trahajo, enn un alcanoe más umplin y un scritiómucho mas humano. Parz niestao amable loctor no pasara desapertibido que ae requerinan nuevas hahilidades directivas y del persnenal para que pueda darse este paso. como fa polivalencia, la actitud favorable hacia

el cambio, nuevos esquemas de tespoasaloilidad y vontrol, empoderamiento, entre otras, sobre algunas de lai evales volveremos más adelante.

En ef esquema descrito nos encontramos ante suna organización măs abierta. integrndora y plurifuncional, que ofreve un mayor campo de acciỏn a la peraona, desempeñando uass funciones u otrasde scierdo conlas demandas del mercaulo: Ala vex, se le abre un nuevo camino de desarzollo professonal. Colocamos a la persona - Ia finica que puede ser de verdad capaz, creativa y Bexiblo - en el centm de la actiridad de la empresa. * (3).

En ssma, el ser humana pasa a ser el factur clave en este enfoque, en lugat de un simple ocupante de un eargo, sus aspirucisnes y talentros envientran muer reconocimicato, oportunitudes y exigencias, la variedad y el entiquecimiento en el qué y cómo bacet despinzan a la rutina y la monotonis que casacterizan a quicraes desempeñan durante muchu ticmpo un mismo carge, además de ha sinergia que se adquiere on el aprendizaje $y$ el aporte que se entroga al trabujar en equipo.

\section{LA DIRECCIÓN DE EMPRESAS Y LA NUEVA DIRECCIÓN DEL. PERSONAL}

Tradicjoralmente, las empresas han enfocado man politicas y pràcbicas sobre el personal en términos de costos, lo que en huena medida explica por qued los recortes presupuestales por lo general comienzan por los rubeos de bienestar y eapacitación o ineluso, In fendoncia a sanear las finanzas de la conpresa mediante el recorte de personal y lu disminuición consecuente del valor a pagar por ouncepto de nómina. Pensar y actuar de esta forms impide ver ef taiento humano como un factise competitive clave para fa empresa y limsita seriamente la pointilidad de suóptimo aprovechamiento. Si a lo dicho atoñodenos la costumbre de eperar bajo estandares ea lo que se refiere a la efiescit operativa det personal, nos osesformaremos con cumplir, lo que represeata no avanus y si, covo leroer clemento, laa normas y reglamentos constituyen el eje centrul del control. estaremos juzgando a nuestra gente permanentemeabo y uspararemos a que se amaneje bien na por onnvicción sinn por femor o por evitat problemss y sanciones (ai se me permite, acuấarin la expresiono sno itar papayas), arnectn de kr errado de creet gue el comportamiente de las personas solo sbodoce a fo normativo, cusndo responde también a to afectivo, lo valorativo y to umbicatal, para solo citar algunas variables adicionales.

El panomma esbozado disth mucho de las exigencias que sobre el particular peran sobre las empresas modernas, Si se quiere set competitivo, hay que costar son la mejor Bente, la más preparada, la do mayot creatividad y construir el ambiente para que paedan aplicar y desarrotlar sus saberes, eapacidades y taleatos, generanda innovaciones en los bienes y servicios; asi como mejoras mastitucionales, todo lo cual es poeo probable o de limitada efiencia on el lango plazo si nos stenemos a lu fradicián empresarial descrita. Toena cuerpo entonces el segundo gran impocto de las tendencias administrativas achaales en la gestón humans.

UNA NUEVA DIRECCION DEL

\section{PERSONAL.}

Seîlemos de entrada que lo normal es que primero se definain las estratogias del negocio. pura luego asignar y poner a su servicis los recursos de ta empresa, incluidos los humanos, aun caundo poce participurán en la definición de las mismas, especio restervado para uoos poces en la corgaruzación. El papet def personal se resumis a contribuir al logro de las estrategias y lot objetivos a los que se refieren. Otra cosa es euando desde geatión bumana se contribuye al In definición e implementacrón de las estrategias, dundo al personal no ef carácter de recurso subontuasdo a lis estralegas, ains de actor en eoendinación con is cstraiegia, Fin una u otro esso el rol del írea de personal send diferente, tema que retomatemos at analizat el perfil del responsable de la citada área.

Ne debemis pender de vista que las polities $y$ acciones de personal se enmarcas y guian por la filosoffa de la emptess y por to tanto deben ser coherentes eon los valores, principios, normas, simbolos y demas ciementus que en su conjunto conforman la cultura organizacional, con base en usa relaciós dinámicat que perntiti, adicionalmente a repotaria, modificarla so es preciso anie la realidad competitiva de la empresa, ins demandas del estorno y lan expectativas legitinas de los empleados.

Las empresss modernas vienen trabajando sis politicas y prateticas de peroonal desde tres dimensiones: Esfuerzo en Recursos Humanos, Eficacin Opentiva y Sistemus de Control, pero con un planteamiento que trasciende los evatos de personal hasta llegae 
a la inversión, superando lns estindares mediante el mejoramiento continuo y dande lugar al compromise en vez de las normas, lo que en ningain caso quierc decir que los eostos, los estindares y les normas sean malos, sino que resultan insuficiestes para responder a los retos que el mereado planté.

Cuando hahlumes de inversión es lugar de oostos, estaremos buscando la rasuent do maximizar y no de reducir y nos compronetemes con la evaluscion de la pestion y los resulendos de los recursos destinados. por ejomplo a capacitación, y no oon el control de cifras solamente, buscando seterminat la cportunidad y real aporte de fo invertido. La inversión es dinamica ya que entiende que los aportes del personal estain relacionsdos no solo con lo que actualmente sahen y estía en capacidad de hacer, sino que su contribucién ya ligada ademds a los eneocimientos y eapacidades que potencialmente desarrollen, siendo esencial para ello el apoyo de la organtzación. De hecho, hay que cusdar el captal intelectual; pero sobre todo, aerecentarlo,

Fil medio oompetitivo obliga a que continuamente mejoremos nuestro trabujo, no basta con aleanzar un tope, pues por bueno que éste ses debemon mejorarlo o alguien distinto lo hari y sufriremos las consecuencias. En ainteris, la eficiencia y la productividad hay que mejorarlas permanentemente $y$ no odormirnos en los laureleso Cisfrusando de les miveles alcanzados. Delencrse ya es retroceder. Pam lograr las mejotas desesdas, debemos desarrollar al personal y otsegarle us protaganismo más decidido en lay decisiones que afeetan su ariea de trabajo, En atas a darle transparencia y respaldo a los procesos de mejomamiento, habri de medirse la efectividad de gestićn humana aún a pesar de la complejidad de hacerfo con referencis a temas como motivación, cultura, calidad de vida laboral, ete. Es imperioesa entonces la creación de un sảstema de indicadores de gestiòn y cl usa de hemarnientas oomo el benchmarking que permitan evaluar nuestros proxesces y resultsdos internamente de manera más objetiva y compararlos con otros modelos y empresas, cantando con informakion de insyor calidad para decidir y ayamar en el faturo.

El basar et control en la creación y cumplimicnto de normas es limitade y poco realiata Las normas no pueden prever todos los eventos y satuaciones posibies, máxime en un entomo eambiante, nî tampoeo contituyen un factoe motivacional importante debido s que por su musma naturalexs son abstractas, rigidas, generales 6 imporsonales, cusndo cada ser humano es diferente y bo que requerimos es thexibilidad y antes que actuaciones de carbeter eobligadew, genuinas conductas de convieción y compromiso. En este sentido, el papel de Gestión Hamana radieara en lograr la identificacions y adhesion del personal : la eultura de la organización, modiante una misión - visiòn oumpartida. No olvidenos que para que funcione un sastema de control de este tipo es imprescindible que exista una filosofia sobre las personss que reconozea su autodeterminación, parta de la confiarva ell el wor humano y elijs ef diälogo y el respeto mutuo en lugar de la imposición. He ahi lo realmente dificil de cambiar, porque demanda perdida de poder, desarrollo de nuevas habilidades directivas, vencer el fernor al carnbio, exclusión de la deperdencia expresada en el hocho que los subilternos avandan en el camine a ser pares, metsoc costrol jerirquaice y muevas reglas de juepo en un ambiente farticipativo y grupal, en el que muchos directivos no se sienter cò̀nodos ni beguros.

\section{CARÁCTER DE STAFF}

En nuestro medio ha hecho carreru considerar corno responsable del personal a quaen dirige el departamento, la diviaión o exa general el àrea de recursos humanos. Nada màs desacertado y contraproducente. Cada fefe debe asumir el compromiso de dirigir sus empleados, toda ver que es alli donde se presenta y tealiza la relación de trabajo, Si reflexionamos, existe consenso on las acciones o procesas de gestión humana que desarrollan las campresas, tales comno selecxicin, desarrollo de personal, eompensación, evaluación del desempeî̀, comunicación bienestar laboral, entre atros. Pero sobre quién responde por cllas, ¿tiene sentido que el director de Gestión Humana, que ne es especiafista en producción o mercadeo ses el que esersas a quicin se contrata para dichas ineas, proceda a determinar el tipo y forma de trabajo que ef empleado desarrollark, evalúe si lo hizo bien, to acompañe y apoye darante el tiempo que permanezea en la empresa? Es obvic que no, porque no tiene ni conocimientos ni astoridad para hacerlo. mientrat que los responsables por produceión y menesden si puoden y deben realizarlo, decidiendo con equien quieren tiabajar, facilitando el ajuste entre el emplesdo y su ärea de trabajo, acompañindolo cn st gestión, evaluandolo, focompensandolo por sus logros, desarnollando su potencial, generándole coodiciones y eatimulos propicios para que haga bien su trabajo y lo mejore eontinumente, dändole la carn inclaso en momenirs tan criticos eomo in ferminación de la relacibo laboral. Camo afirma Carlos J. Ssinchex-Runde, af_o que pedimos a un buen mando es extremadamente complejo: que sea tesnicamente 
eompetente es as àrea funcional (ventas, Jopistica, producción, contabilidad, efe.), que asuma in resooesabilidsd poe los resultados de wu unidad, que demoestre cualidades de liferaxgo (viaicen, etica, carisms...), que ayode al desarnollo protesional de sus isubordinados. y que genere entusiaamo-motivacion- entre stas colaboradoreso (4)

En el maron descrito, gara qué entoncos un trea de ponomal? ycase part que onmpita con los jefer o los suplante? En un moments dado, podris euestiosar la gerencia que sentido tiene dupticar esfuerzos y pagar dos veces por lo mismo. El papel de Gestión Humana radicari entonoes en ayudar. colaborar, aseserar, oricatar y poner a disposicion de los jefes las herrmmientas qoe tequieran para cumplir su labor eon ef personal. De rosponder por las personas do manera directa, pasa a ser un área de soporte y apoyo, to que marca el tereer impacto:

\section{CARACTERSTAFE.}

Es facil eacribir lo anterior, pero extremadainente complejo vivirlo ante la isentalidad adversa a bo propuesto, el inmenso trabsojo y riegzo que significa para los demás directivos acturer baio esta peripectivas $y$ todo un seumulado historieo que poir inercia presonta a defar las onas taf como estin. No obstante, if miramos con detenimiento. encontraremon que sं Gesticon Humana no posee responsabilidad de lines, se puede reducir sensiblemente sa extructura, alivindo los eostoa $y_{+}$por qué no, pensar on el cutsourcing como mecanismo vilido parn la prestación de bos servicios que ie son propios. Pot atra parte, todo cuanto se logre en el deatrollo de lideres (jefes) integrales redundari en un mejor mubiente ve trabajo, posibilitara un optimo aprovechamiento de la fucrea latoenal que incidirà ensu dencmpetio y productividad, facilitarí y reducirà los tiempos de toma de decisiones sobre el personal, disminaira rivalidades y oonflictos al intenor de la coganización y se acabarin Jos señalamientos tratando de eludir la responsabilidad (es que el personal i..en que el jefe de producción ... ). constituitá un mejocarsiento personal y profesional pars kos directivos y aedi vital purnel trahajo en equipe dentro del esquema de red, como veremos posteriomente

\section{Polivalencia $\mathbf{Y}$ TRABAJO EN EQUIPO}

Los factores competitivos obtigan a tribsiat en equipo, aprovechando las competencias individuales de sus miembros y enatando con se disposición al aprendiraje $y$ desarrello de nuevas capacidades y al desemipeón en tareas diveras y cumbiantes, de tal suerie que la valia de un empleado no se cifis a un etreg. sine a múltiples funciones y actividsdes a las que se adapta $y$ ejecuta con facilidad sleanzando buenos resultados. Un cuarto impocto sorá el de ALCANZAR LA POLIVALENCLA Y EL TRABAJO EN EQUIPO,

Aunque hemos expuesto algunos de las beneficios de las astens de trabejo en lugar del pueste de trabajo y del trabajo en equipo sobre el desempeño individual, es probable que los mismos funcionarios resistan el cambio, ante ol descosocimiento de sua capacidades para actuar y sobresalit en un grupo, las debilidades que posesn para desempeflarse en tareas diferentes a las de su eargo, ei arduo trubajo que les espera, la incomodidad de encointrarse hacieado algo que los desagrade o con personas yue preferifian evitar, et depender a retrihoción y hasta la estabilidal en ef empleo no solo de si mismo. sino del trabajo de otros y pot un factor eulitural que nos invita a in competencia antes que a la colaboración y al individualismo antes que a is tunione. Aate hechos come estos, is labor do mostrar kos beneticios, preparar para ef tambie, desartollar las noevas capacidades y lograr la transformación de less barrena: culturales reaumsen las retos en maleriu de gestion humana, unotando que deberán tanbie'n replantearse los procesos de personal pues es apenas lógivo que al buscar diferenves competencias en ef perronal, kendremos que geleccionarlos con base en parámetros distintos, remunerar su trabajo inciuyenulo ademis del exfuerzo individoal la labor colectiva, evaluarlo tamhièn oon referencia al grups, replantear las planes de currera y lo relativo a la promeción por meritos exchasivamente individuales que represesta suhir en un organigmama, clorgar poder para decidir, tecursos suficientes para laboraf, rediseñar el sistema de control, es decir, redefanir assi por complicto suestro actuar.

\section{ADMINISTRAR LA RELACIÓN ENTRE TECNOLOGIA Y EMPLEO.}

El veloc y gigtatesco avanee delas testoologias de información ènéde de mahera directa en la gestion humana, tanto come facilitador -de herramientas que permiten lacee de maners más eficiense ef trabajo, como automalozando los prosesos, desplazando en ecasiones la mano de obta o cuando menos haciendo necesaris una mayor cuatificación de is misms o sa reubicaoibn, La tecpologia incide entonces 
en las condiciones y molndos de trabajo y gracias a ella han surgide nuevas formas de empleo (teletrabsjo) y de empreas (corponaciones virtualen, ofieina móvil). Hoy, un trabajador que ne posea canocimictitos apropiados con las debidas habilidades y destrezas para ef manejo del oumputador y los diversos softwares, serti exclaido del mereado laboral. Las empresas se organizan en red y boena parte đe su información y tegocios la realizan pơ esta víz razỏn que dernanda de su gente el dominio en este campo. La winvasións de le iecnologia impone rediseñar los cargos ylo las áreas de trabajo para garantizar su adeesada utilussción y excluir aquellos pasos del proceso que yu no fiesteo sentido porque no se efectuatán manualmente o porque el tiempo requerido es mucho menor, pudiendo agregarse tareas al personsl existente para evitar que quede ocioso e incida nocivamente en la eficiencia y los costos. En este panto es pertinente destacar que low mandos medios son los más gravemente afectados puesto que is tecosologia efectuari muehos procesos de control, que era en buens modidi to que justificaba aus cargos y porque anve el acceso mosevo a la infonnación, se diluyen las telaciones jerírquicas y se propende por las estruchuras planas con lidecazges bassdes en la stituación particular, no centrados en personas determinadas, El sitio de trahajo se ve tambièn afiectado porque en muchos casos, conectado desble el hogar, un trabajador podrá desempeñar sus labores y no se requerina su presencia física en la empresa, dotsndo de flexibibdad ta relación laboral, asi eomo al nivel ejecutivo se le facilitan sus desplazarnicntos fluera de ls empresa al poder permanocer en contueto cos ésta desde eualquier lagar del planeta y, lo que resula mejor aùn. llevando consego la información nevesaria o estando en capacidad de obtenerfa en instantes, agiledindose los negocios y soportando su dimerisión global.

Gestión Humani debe aptovechar las facilidades que la tecnologia brinda para la selección de personal, consultando ofertas de trabajo en intemet, por cjemplo, o anunciando en ella sus requerimientos de personal, o dischiando planes de capacitación en modalidad virtual. con las veatajas de menotes castos, mayor cobertura y ssitcronismo (no interferir en el borario de trabajo), diseñando e impicencatando, o bien adquiriendo softwares para la administración del personal y nómina, es decir, en pocas palsbras, asimilando y utilizando al máximo las tecrnologias de la información que estén al alcance de la empresn.

En alguna medida el auge de los sistemas computacionales esustituiri la relaçón tabocal interhumana por una relación hombreimáquina que rompe las posibilidades de comunisecsón social a traves del trahujou (5), a la vez que puede acentuar el eomponente rutinsrio de is laber. generar nuevas tipologias de enfermedades profesionales y, lo que veria alarmante, el aislamiento del trabajador, elementos que doben analizarse a profundidad y desde fodo punto de vista controfar

Paradójicamente, la informatica configura una oportinidad para biberar al hombre de trabajos futinarios y subalternoa potenciando su creatividad, climinando via automationción de los procesos una sene de riesgos en el trabajo y generando esposcios pura actividades diferentes y enriqueotdoras que van desde lo labonal, pasando pot bo inteloctual hasta la recrentivo. fTodo depende del manejo que le demes a las tocnologins de la información!

Gracies a la tecnologia se subeontratará eada vez un espectro más amplao de selsvidades que realiza in organizacion y que no estin ligadas a la esencia del negocio, en detrimento de la fuerza de irabapo. Ante esta realidad, debemos preparar al personal para que aprenda in trabajar desde ufuera» de la organizactón y sea conscienie de la ilusión que representa en los tiempos modemos la estahilidad lahoral ligada a uni sola empresa.

Los anteriotes postulados configuran el quinto impacto CONCTUAR TECNOLOGIA Y EMPLEO.

\section{FLEXIBILIZACIÓN LABORAL.}

El moreado demanda continuamente innovaciōn y adaptación velog de lss empresas a sas requerimientos, to que es posible si la organización es flexibie, ya que por defísición, lo rigido es inamovible, inallerable, bende a la permanancia y cosservaciob del estado de tosas tal cial como cuanido se cren. El eambio, palabea màgies y compleja, de uso más que frecuente en el umbiente empresarial, lleva en su seno la noción de Aexibilidad, porque pasar de un estsdo a otra plantea transformar, adaptar, variar y, ante todo, comprender que nads es estricto y riguroso, que todo puede pensarse y hacerse de maiser: diferente y que ef sentirse intocable o inalterable resulta una actitud un insolita comso nociva en nuestros dins.

En la medida que se pueda eseoger entre varias alternativas y h empresa y sus empleados no se vean limitados a una sola upcóón en 
el docidir y el actuar, estaremos denitro det inareo de in fexibilizacion.

En cl canipo laboral sedefine la tlexibilización como ula capacidad de los individuos es la coonornia y en particular en el morcado de trabajo de remunciar a sus costumbres y de adaptarse ar nuevas circuastanciase o como la neapacidad de adsptación del régimen sormativo y del juego de las instituciones a la voluntad unilateral o biluteral de las partes de la relacionn de trabajoo (6). La legisiación ceroea rigida, ha eedido, dando lagar a que las relacianes laborales re rijan en mayor modidn por In voluntad y autonomia de las partes y conternplando en us propios preceptol institucioos ontho la centrutación a temnitio fijo, a tiempo parcial, el trabajo de medio tiempo, el salaris integral, el desmonte de figuras como el reintegro y la retroactividud de las eesantias, ea sums, una serie de Gisposiciones que modificaron instituciones del derecho luboral y privilegian los acuerdoa eatre las panes (empleadot - trabajador) en temmines de fa jormada laboral, ef carácter salarial o no de los pagos y el tipo de contrataciồn, como stus factores destacados. La reforma laboral propuesta en nuestro psis profundiza en la tlexibilización y propone compensar boras extras y trabajo explementario con tiempo de vacaciones en lugar del pagor en disero eon los reesfgos que actualmente aperan, ta posabilidad de los ecentritos de trabajo por horas en los que no se remunerari el tiempo tabotado en dominicales y festivos compo actualmente se bace y que llevarian a que una sola penona pueda labarar, con contrato de trabajo, para varis entidades kimullinneamente, evitre otros aspectrs. Es claro que si pretendemos que las empresas respondan àgilmeate a los cambios, deben poder bacerlo con fodus sus revursos y afectando todos mus componentes, inchidas los empleados, no entendorlo asi forraria el sostenimienta de coiffas elevedos, de relsciones despastadas o innecesarias, de viejos eatilos de hacer las cosns no compatiblea con las expectativas actuales y se derivatia en posiciones de franca desventajar ante in competencia ahora global. Un sexto impacto se vislumbra ain que pueda impedirse: LA FLEXIBH.IZACIONDE LAS

\section{RELACIONESLABORALES.}

Desde luego que en principon la parte mas favorocida es la empresa debido a que posee los medios y puede, bajo la apariencia del acuerdo, presionar a, mejor aún, imponer condiciones, pero no se trita de discutir lo afortunado o inconveriente de este hecho, sino de aceplar su reabded y proparamas para siu usa, desarrollando habilidudes de nejuciacion Y preparándonos para vompetir, como empleados, es un mundo mas abierto, competbdo, incstable y de mulbhad do opeicoes a la hon de eonfigurir Is relaciós laberal.

Nuses antes oome ahora toma sentido la expresióa segun el eual «EL QUR NO CAMBIE, LO CAMBIAN",

Hernos pecteofido resaltar los impactos más relovantes que las tendenciss actuales goneran en la gestión humana al interior de las empresal, siendo conseientes del tratamiento sintétion que por razones de espacio y la natumaleza de este eserito se le han dado, en defecto de sematicas tan interesantes para peotundizar como el sistema de remuneración por competencias, el dischio e implantación de las areas naturales de trabajo, el trabajo virtual y las ofganizaciones. virtuales, las buevos sistemas de control y de participacion, el enpital inteleetual, ete, pero si hemos motivado al lectos a reflexionar sobre la que nas espera a la vuetta de la esquina $y$. consecuentemente al estudio y profundización de estas temáticas. aportándole una lur para cl camino, habremos cumplida en buena medida nuestro objotivo No obstante, seria imperdoable no destinat al menos unas poosi lineas para abordar el perfit del responsable del áres de personal, acorde con lo hasta ahora expresado:

\section{EI NUEVO PERFIL DEL DIRECTOR DE GESTIÓN HUMANA}

Conservando in que es apenus obvo, como el Dominio de sur Area Especifien: La Núministracsón de Personal (o más exactaunente la Difeceico de Persinal, porque los recurnos se adminiatran, pero las persoests, que no soe cosas, se dirigen); Excelentes Relaciones Interpersonales, Fitulo Protesional y las Condiciones Esicas de rigor, nos atreyemos a formular la siguente caracterivacióñ:

Conocimiento de los Procesos Operativos, csencial para enteader qué hace la gente, poder diseñar las áress aaturales de trabajo, formar y desarrolat equipos de alto descmpeño, coninbuir a les resultsdos de la empresa, proponer movimientos de persinal, efe.

Sentide Fooniention de ss Función, lo que implica conocimiento y utilizacicon de la Estadistica, las Matematicas, la Economiay las Finunzas. Recuéndese que se requiere medir la gestión y evaluar resultados pari determinar la eontrihuciós (valor ayeregado) del àrea de gestión hurnana a la orgammeión. 
Pensamiento Estratégico, para dimensionar ol negocio, definit entrategias y ver emociente del papel del personal come factor elave de competitividad. Exige, ademàs del manejo de técnicas prospectivas, elementos de mercadeo.

Dominio de un Segundo Idioma, que resulta definitivo en un ambiente global, de preferencis et inglés.

Cspacidad de assimilación y utilización de las tecnologias informáticas y los avanoes tecnologieos.

Habilidades Directivas, como Trabajo en Equipo, Toma do Decisiones, Conmunicación, Negociación y Liderazgo.

Tolerancia a la Frustracion y Capacidad para Trabajar bajo Presion.

Alta Motivación al Logre

Experiencia Profesiotal y Educación Avanzada, de preferencia a nivel de Magister o Doctorado, si bien este requisito depende del tipo de empresa y seria pócticamente inalcanzable para empresas de oorte mediano, pequetio o microempresas.

Urge elevar ef nivel del área y do exigeneia para quien la diripe, ante la complejidnd e importancia de las labores a sa carpo que reclaman un profesionat de excelente nivel y sobre todo, integral

\section{CITAS}

(1) Faente: RJCARD, Jnan Enric, nNuevas Formas Organizativas: Funciananientos Publicado en Cómo Prepararse para las Organizaciones del Futuro. Bibliotees IESE de Gestión Empresarial. Universidad de Navarra. Ediciones Folio, Barcelona, 1997.

(2) GOMEZ, Sandalio, La Persona y el Trabajo en la Erapresa del Siglo XXI. Biblioteca IFSE de Gestion de Empresas Ediciones Folio, S.A. Barceloos, 1.997.

(3) IDEM.

(4) SANCHEZZ RUNDE, Carlos J. Dirección Estrabégican de Recurnos Humanos, Bibliotess IESE de Gestion de Empresas. Ediciones Folio, S.A. Barcelona, 1.997

(5) JOYANES, Luis, Cibersociednd. Los Retos Sociales ante un Mundo Digital. Editorial MeGraw-Hill/nterameticana de Eapañia, S.U. Madrid. 1.997.

(6) ALVARFZ PEREIRA. Carios. Fexibelizacion de la Nematividad Laboral Revista.Actualidad Laboral Nro. 58. Esitorial Legis Santa Fe de Hogoth. 1.993 